3. Two hypersensitive patients were desensitized, and successfully operated on for immature cataract.

4. The test is necessary only in the following types of patients :

(a) Those with immature cataracts. If the test is positive they should be desensitized or operation delayed until the cataract is mature.

(b) Those with traumatic cataracts. Every effort possible must be made to remove the lens matter by operation.

(c) Those failing to develop good vision after a previous operation on the other eye, and particularly those with a history of long continued pain and redness.

\title{
F. A. Williamson-Noble.
}

(3) Gruss, Dr. Robert.-On stovarsol. (Zur Frage des Stovarsols.) Wien. klin. Wochenschr., March 27, 1924.

(3) Gruss reports a case of primary chancre treated with stovarsol. When the patient was first seen spirochaetae were present in the chancre but the Wassermann was negative. In the course of seven weeks the patient was given altogether fourteen grammes of stovarsol - by the mouth. At the end of this period the chancre had healed up, but there was a generalized papular syphilide and the Wassermann was positive.

\section{A. H. LEvy.}

\section{BOOK NOTICES}

The Relative Position of Rest of the Eyes and the Prolonged Occlusion Test. By F. W. Marlow, M.D., etc. (Syracuse, N.Y.) Philadelphia: F. A. Davis Co. 1924. Price, \$2.50.

For several years past, in communications to various medical journals, the writer of this monograph has advocated the employment of the prolonged occlusion test in the examination and determination of errors of ocular muscle balance. Nevertheless, the test is, so far as we are aware, but little known or utilized in this country. In the book now before us, which is based on his experience in 700 cases, Marlow gives a clear and well-expressed exposition of his views, a detailed description of his method of examination, and the results of its employment during a long series of years. The title of the book seems open to criticism; the "Relative Position of Rest" has pride of place, but only two pages are devoted to its consideration. These two pages, however, contain statistics which are probably unique; they give the relative 
position of rest of the eyes of 700 persons as determined by the prolonged occlusion test; in every case the influence of errors of refraction was eliminated. In only 24 of the whole number was the relative position of rest one of parallelism.

The prolonged occlusion test consists essentially in complete occlusion of one eye for a much longer period than is posssible during a clinical examination. In the author's words : "it is nothing but a screen test extended in point of time." "Theoretically . . . . the test should last until stability in the position of rest is attained, that is until the findings are essentially the same from day to day; but as a rule a week's occlusion will furnish valuable information."

The results obtained by Marlow in his 700 cases are shown in tabular form; in table 10 there is a summary of the general results of the test, which merits attention. Of the total of 700 cases, 154 showed orthophoria before occlusion; after occlusion this number fell to 13 ; in percentages these figures are 22 before, and 3.4 after occlusion. These figures are remarkable and strengthen the reviewer's belief that errors of ocular muscle balance sufficient to give rise to symptoms occur with greater frequency among the inhabitants of the United States than among residents in this country. It would be interesting to know the relative number of males and females in the author's collection. The tables give no indication, but there is one statement in the letterpress which suggests that the greater number of his patients were females.

Marlow writes with the conviction of one who has tried and proved the value of this test of which we believe he is the originator. It has, he says, "yielded valuable aid in the elucidation of many otherwise obscure problems."

His arguments, coupled with the results he has obtained, shown in the tables and recorded in the letterpress, indicate clearly that his method is one deserving a careful trial by ophthalmic surgeons who are not already familiar with it.

The book is well printed, and is almost free from typographical faults. It is provided with a table of contents and an index.

The Ophthalmoscope and How to Use It, with a Chapter on Diplopia. By A. Freeland Fergus. Lonidon: J. \& A. Churchill. 49 pages with 17 Illustrations. Second edition. 1924. Price, 3s. 6d.

We published a notice of the first edition of this book in the February number of this year. The only addition of any importance consists in a statement conveying the author's views on the more correct way of regarding minus lenses as positive and plus lenses as negative as done by physicists. We must admit 
that strictly speaking this would be more correct, but we fear that the conventional use of the terms by ophthalmologists is of too long standing to be altered for theoretical reasons.

We must congratulate the author on the necessity for a second edition so soon after the first, as this must indicate that the book has been found to supply a want.

\section{CORRESPONDENCE}

\section{MINERS' NYSTAGMUS}

To the Editor of The British Journal of Ophthalmology

SIR,-I am inclined to agree with $\mathrm{Mr}$. A. S. Percival of Newcastle-on-Tyne that in a certain percentage of cases there is a predisposition to the disease from childhood. I am also of opinion that a condition may arise in the eye in later life which tends to induce the condition.

Appended are notes of two cases sent to me for examination on July 20, 1921, which may prove of interest.

1. Richard C., 55, coal miner, residing at Tyne Dock, County Durham. At work last on October 19, 1917. Complains of blindness, giddiness, pains in the eyes, the forehead, and back of head. Has good health otherwise. Eats well and sleeps well. Smokes two ounces of twist tobacco per week. Does not chew tobacco. Does not read. Can read a certain amount with glasses, but does not do so because of fatigue. Has slight nystagmus. Fundi healthy. Commencing cataract. Some central opacity in each lens, more dense in right one. Is hypermetropic.

R.V. 6/60 with $+2 \mathrm{D}$ sph. $6 / 36$.

L.V. $6 / 64$ with $+2 \mathrm{D}$ sph. $6 / 12$.

In this case the nystagmus was distinctly slight. I think he probably had changes coming on slowly in his lenses for a long period. Of course, he was unfit to go to work in a mine again.

2. Thomas Y., 44, miner, Tyne Dock, County Durham. At work last in October, 1914. Complained at that time that "everything was going round and round," also dizziness. When got down the pit had to sit down for a quarter of an hour before able to see the road he had to take. Was very bad on coming out of the mine. He started work in a mine when 13 years of age, and was there until 20. After that was away from mining for 14 years. and worked at filling grain and iron ore. After this he worked in a coal mine for about four years and then developed nystagmus. 